\title{
Research Paper \\ The Effect of Warm Foot Bath on the Consciousness Level of Patients With Head Trauma
}

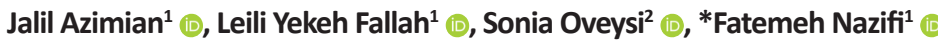

1. Department of Critical Care Nursing, Faculty of Nursing and Midwifery, Qazvin University of Medical Sciences, Qazvin, Iran. 2. Metabolic Diseases Research Center, Qazvin University of Medical Sciences, Qazvin, Iran.

\begin{tabular}{|l|l}
\hline $\begin{array}{l}\text { Use your device to scan } \\
\text { and read the article online }\end{array}$ \\
Citation Azimian J, Yekeh Fallah L, Oveysi S, Nazifi F. The Effect of Warm Foot Bath on the Consciousness Level of Patients With Head \\
Trauma. The Journal of Qazvin University of Medical Sciences. 2020; 23(6):514-525. https://doi.org/10.32598/JQUMS.23.6.4 \\
doisttps://doi.org/10.32598/JQUMS.23.6.4
\end{tabular}

Received: 20 Jun 2018 Accepted: 15 Sep 2018 Available Online: 01 Feb 2020

Keywords:

Consciousness, Head trauma, Warm foot bath

\section{AB STRACT}

Background Head trauma can cause decreased consciousness level and reduced ability to respond to the environment, leading to impaired cognitive, perceptual and sensory functions in people. One of the techniques that can help improve the brain function is warm bath.

Objective The aim of this study was to determine the effect of warm foot bath on the level of consciousness in patients with head trauma.

Methods This is a clinical trial using control group conducted on 50 patients with head trauma having Glasgow Coma Scale (GCS) score of 5-12 admitted to the intensive care unit of Shahid Rajaee Hospital in Qazvin, Iran from June to December 2017. They were selected using a convenience sampling method and randomly assigned into two groups of intervention and control. Intervention included a warm foot bath for 7 days, once a day for 15 minutes, and their GCS score was recorded before, one hour and three hours after the intervention. Data were analyzed using repeated measures ANOVA, chi-square, t-test, and descriptive statistics (mean and standard deviation).

Findings The mean consciousness level of subjects in the intervention group was significantly higher than in the control group after warm foot bath $(P<0.05)$. Three hours after warm foot bath, the mean consciousness level was $9.32 \pm 1.86$ in the intervention group and $8.44 \pm 2.48$ in the control group which was significantly different $(\mathrm{P}=0.005)$.

Conclusion Warm foot bath can increase the level of consciousness in patients with head trauma.

\section{Extended Abstract}

\section{Introduction}

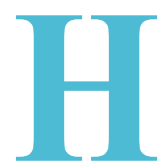

ead trauma is one of the major problems in the world. The annual incidence of head trauma in Tehran is estimated 144 per 100,000 people [1]. According to the World Health Organization, head trauma will be one of the leading causes of death and disability by 2020 [2]. One of the most important consequences of head trauma is the change in consciousness level, which can lead to coma and, as a result, several complications [6]. Sensory deprivation is one of the side effects that threatens patients in intensive care units by reducing the consciousness level [8]. Since sensory stimulation is one of the basic human needs [2], in order to facilitate the recovery process and prevent sensory deprivation after a brain injury, an early

\section{* Corresponding Author:}

Fatemeh Nazifi

Address: Department of Critical Care Nursing, Faculty of Nursing and Midwifery, Qazvin University of Medical Sciences, Qazvin, Iran.

Tel: +98 (28) 33321478

E-Mail: fateme.nazifi@gmail.com 
sensory stimulation program can be useful [9]. This study aimed to examine the effect of warm foot bath on the consciousness level of patients with head trauma.

\section{Materials and Methods}

This is a clinical trial using control group conducted on 50 patients with head trauma having glasgow coma scale (GCS) score of 5-12 admitted to the intensive care unit of Shahid Rajaee Hospital in Qazvin, Iran from June to December 2017. They were selected using a convenience sampling method and randomly assigned into two groups of intervention $(n=25)$ and control $(n=25)$. First, the demographic and clinical characteristics and the GCS score of patients were recorded. Then, the control group received routine care, while the intervention group, in addition to routine care, received warm foot bath from the ankle to the toes for 7 days, once a day between 4-6 PM for 15 minutes using a foot spa massager (EmsiG FW328, China). Their GCS was recorded again one hour and three hours after the intervention. Data were analyzed in SPSS V. 22 using repeated measures ANO-
VA, chi-square, t-test, and descriptive statistics (Mean $\pm \mathrm{SD}$ ). The significance level was set as $\mathrm{P}<0.05$.

\section{Results}

The mean age of the patients was $38.96 \pm 13.52$ years in the intervention group and $38.56 \pm 14.47$ years in the control group. Of 50 patients, $76 \%$ were male and $24 \%$ were female in both groups. The causes of head injuries were car accident $(76 \%$ in the intervention group and $68 \%$ in the control group), falling (20\% in both groups) and motorcycle accident (4 and 12\%) (Table 1). The mean level of patients> consciousness at the beginning of the study (first day of the study) was $8.28 \pm 1.83$ in the intervention group and $7.72 \pm 1.94$ in the control, but it was not statistically significant based on t-test results $(\mathrm{P}=0.254)$. Three hours after the intervention ( $7^{\text {th }}$ day of the study), it became $9.32 \pm 1.86$ in the intervention group and $8.44 \pm 2.48$ in the control group, which was significantly different $(\mathrm{P}=0.005)$.

Table 1. Demographic and clinical characteristics of patients

\begin{tabular}{|c|c|c|c|c|}
\hline \multirow{2}{*}{\multicolumn{2}{|c|}{ Demographic and Clinical Characteristics }} & \multicolumn{2}{|c|}{ Mean $\pm S D /$ No. } & \multirow{2}{*}{ Sig. } \\
\hline & & \multirow{2}{*}{$\begin{array}{c}\text { Intervention Group } \\
38.13 \pm 96.52\end{array}$} & \multirow{2}{*}{$\begin{array}{c}\text { Control Group } \\
38.56 \pm 14.47\end{array}$} & \\
\hline Age & & & & $0.954^{*}$ \\
\hline \multirow{2}{*}{ Gender } & Male & 19 & 19 & \multirow{2}{*}{$0.972^{* *}$} \\
\hline & Female & 6 & 6 & \\
\hline \multirow{3}{*}{ Causes of head trauma } & Car accident & 19 & 17 & \multirow{3}{*}{$0.914^{* *}$} \\
\hline & fall & 5 & 5 & \\
\hline & motorcycle accident & 1 & 3 & \\
\hline \multirow{2}{*}{ History of Craniotomy surgery } & Yes & 17 & 16 & \multirow{2}{*}{$0.8170^{* *}$} \\
\hline & No & 8 & 9 & \\
\hline \multirow{2}{*}{ Respiratory problems } & Yes & 18 & 23 & \multirow{2}{*}{$0.085^{* *}$} \\
\hline & No & 7 & 2 & \\
\hline \multirow{2}{*}{ Upper extremity fracture } & Yes & 11 & 8 & \multirow{2}{*}{$0.093 * *$} \\
\hline & No & 14 & 17 & \\
\hline \multirow{8}{*}{ Diagnosis } & & 11 & 9 & \multirow{8}{*}{$0.171^{* *}$} \\
\hline & Concussion & & 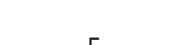 & \\
\hline & Intracranial hemorrhage & 6 & 3 & \\
\hline & Subarachnoid hemorrhage & 4 & 3 & \\
\hline & subdural hemorrhage & 2 & 4 & \\
\hline & epidural hematoma & & A & \\
\hline & \multirow[t]{2}{*}{ Cerebral edema } & 0 & 4 & \\
\hline & & 2 & 0 & \\
\hline
\end{tabular}




\section{Discussion}

The results of this study showed that although the mean GCS score of the patients in the two groups was not significantly different on the first day of the study, but on the seventh day, the mean GCS score in the intervention group was higher than in the control group. Mandeep (2012) using early intervention of coma arousal therapy in traumatic head injury patients, also examined for changes in the levels of consciousness in the intervention group on the first to 14th day, which had an increasing trend and there was a significant difference between the results on first, 7th and 14th days. This is consistent with our results. In our study, the increase in GCS score of the intervention group showed a significant difference from one hour after the intervention to the third day (the end of study). Hosseinzadeh et al. [6] also reported a significant increase in the level of consciousness of the patients from the third day of the study compared to the controls, but in the study by Oh and Seo [30], patients had significant changes in their level of consciousness 2 weeks after the sensory stimulation. In their study, the intervention was performed for 5 days a week, while in the present study, the intervention was conducted for 7 consecutive days. The reason for this difference may be due to the continuous form and interruption of the intervention.

Overall, it was concluded that, by sensory stimulation programs such as a warm foot bath, we can help increase traumatic head injury patients' level of consciousness and reduce its complications. Proper sensory stimulation increases consciousness and can improve the prognosis of the disease. Therefore, the use of complementary therapies such as warm foot baths is recommended as a common clinical method.

\section{Ethical Considerations}

\section{Compliance with ethical guidelines}

This study was approved by the Research Ethics Committee of Qazvin University of Medical Sciences (Code: IR.QUMS.REC.1396.26). It is a registered clinical trial (Code: IRCT20180731040658N1). Prior to study, permissions were obtained from the Qazvin University of Medical Sciences and the study objectives and methods were explained to the patient companions and their informed consent was obtained. They were assured of the confidentiality of their information.
Funding

This article is taken from the Ms. thesis of Fatemeh Nazifi from Faculty of Nursing and Midwifery, Special Care, Qazvin University of Medical Sciences.

\section{Authors' contributions}

Conceptualization, design, methodology, review, supervision and project administration: Jalil Azimian and Leili Yeke Fallah; Editing and review: Leili Yeke Fallah and Fatemeh Nazifi; Data analysis: Sonia Oveysi; Writing, data collection, intervention, funding acquisition: Fatemeh Nazifi.

\section{Conflicts of interest}

The authors declared no conflict of interest.

\section{Acknowledgements}

We would like to express our gratitude to the Vice-Chancellor for Research of Qazvin University of Medical Sciences, officials, professors of the Faculty of Nursing and Midwifery of Qazvin University of Medical Sciences and the staff of Shahid Rajaei Hospital in Qazvin who helped us in this research. 


\title{
تأثير حمام ترم يا بر سطح هوشيارى بيمار ان ضربه به سر
}

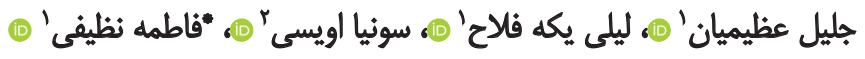

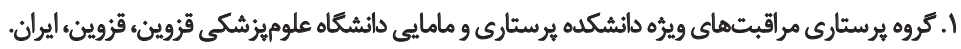

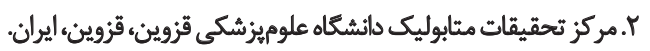

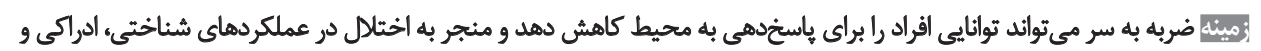

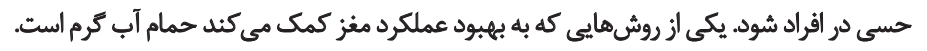

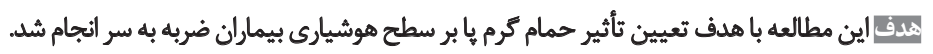

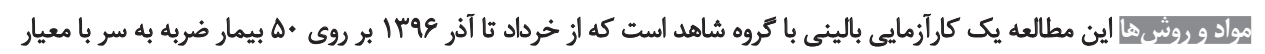

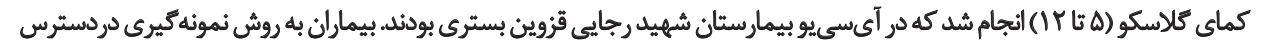

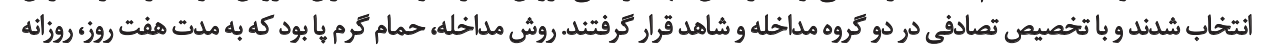

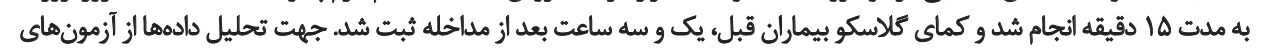

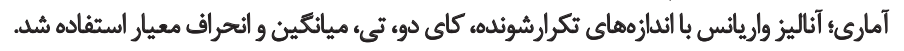

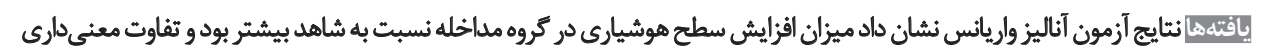

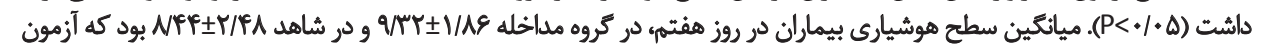

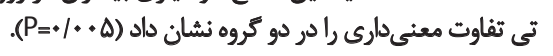

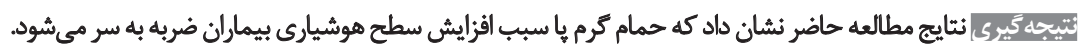

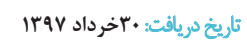

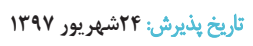
تاريخ انتشار: Tا بهمن

درواقع در كشور ما سوانح و حوادث بـ از بيمارىهاي قلب و مرومين

مelot

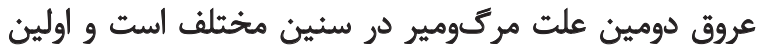

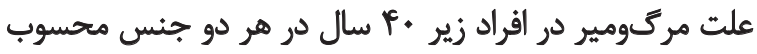

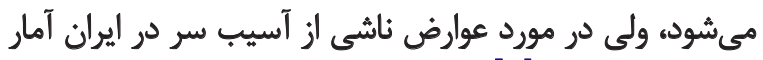

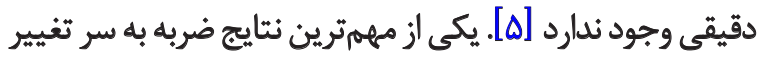

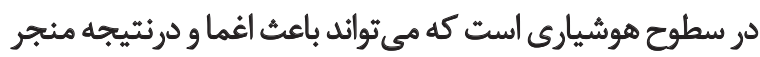
به عوارض متعدد شود [عارئ هوشيارى به طور مستقيم قابل اندازمخيرى نيست وبانشانههاى

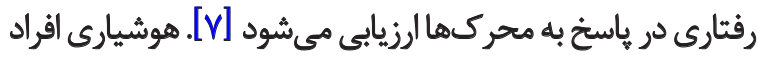

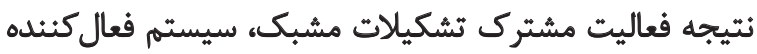

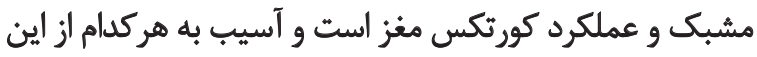

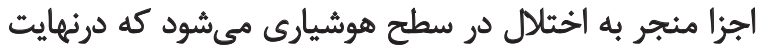

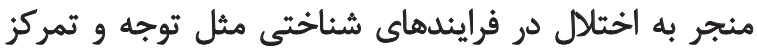

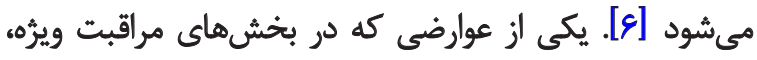

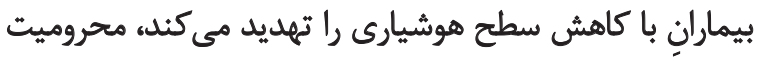

ضربه به سر يكى از مشكلات عمده در سراسر جهان است.

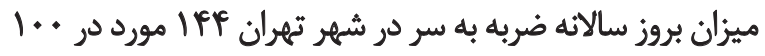

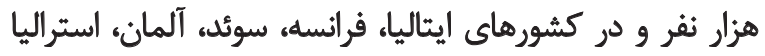

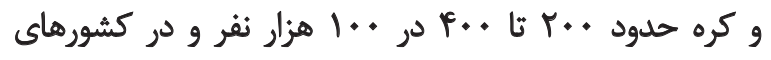

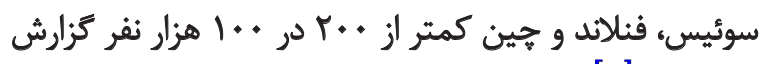

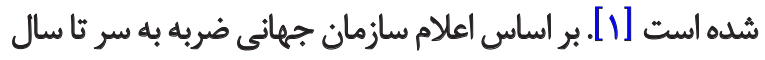

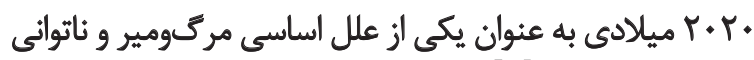

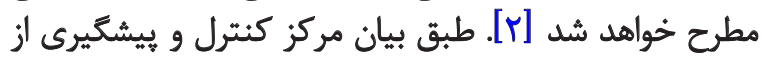

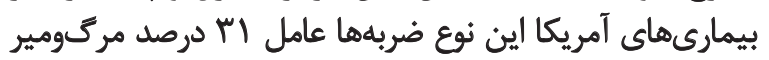

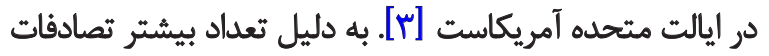

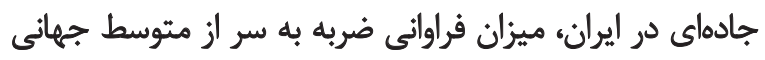

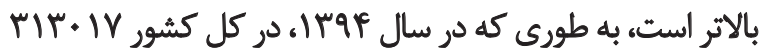

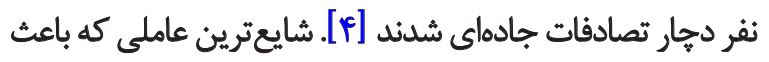

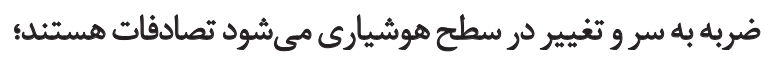




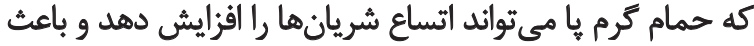

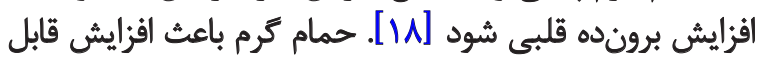

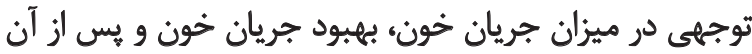

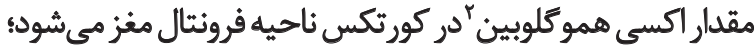

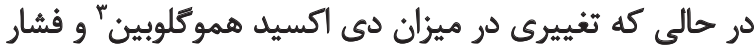

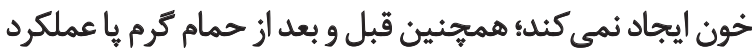

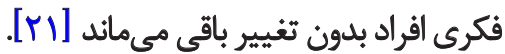

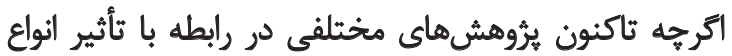

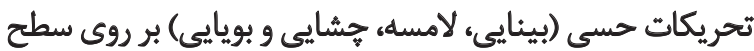

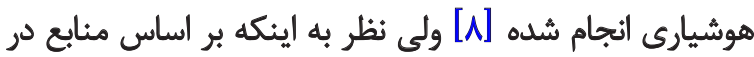

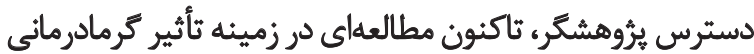

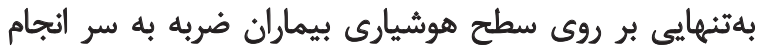

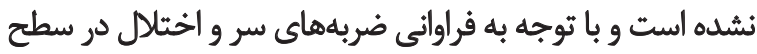

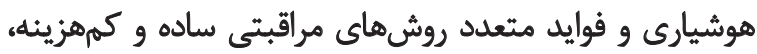

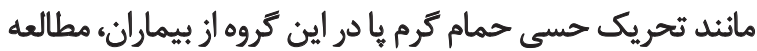

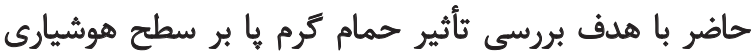

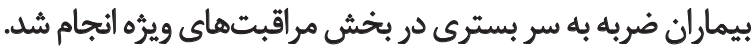

\section{(1)}

اين مطالعه، يك كارآزمايى بالينى با كروه شاهد است كه از

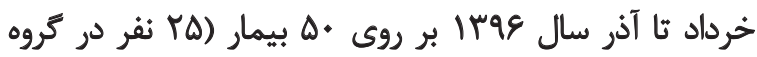

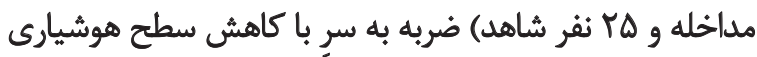

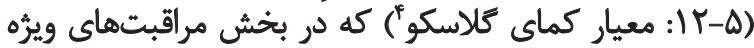

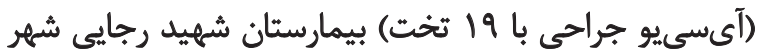

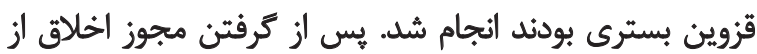

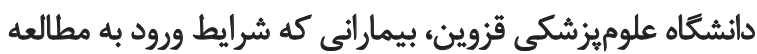

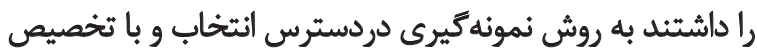
تصادفى در كروه شاهد و مداخله قرار كرفتيند. معيارهاى ورود به مطالعه شامل بيماران ضربه به سر سر داراى

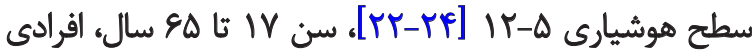

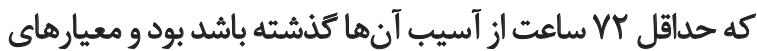

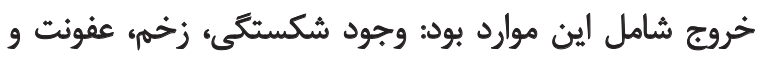

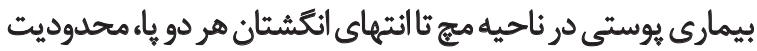

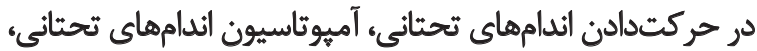

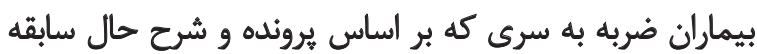

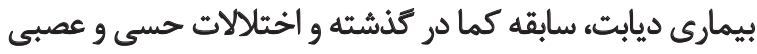

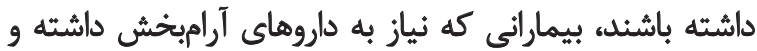

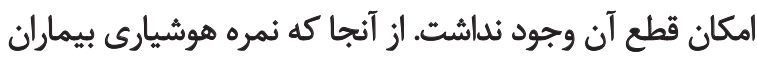

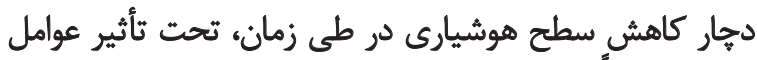

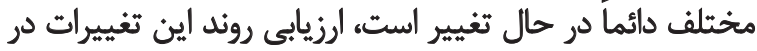

\section{Oxyhemoglobin}

حسى است [N]] از آنجايى كه تحريك حسى يكى از نيازهاى

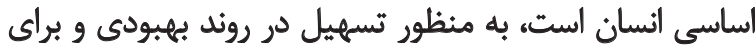

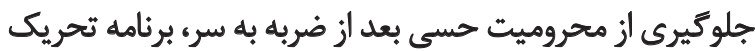

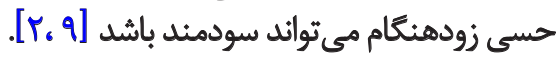
فرايند بازسازى و بجبود مغز كه هيلاستيسيتى ناميده مى بشود،

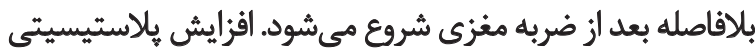

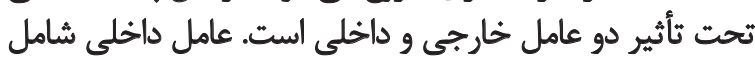

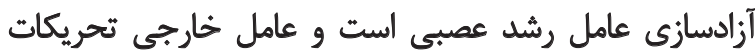

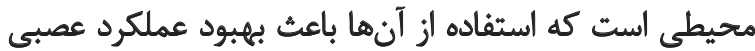

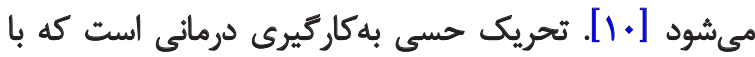

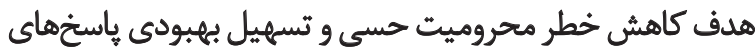

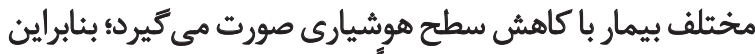

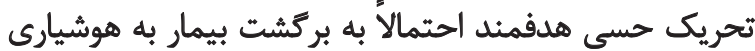

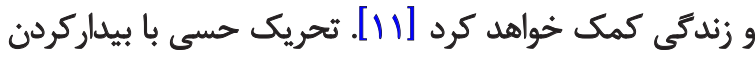

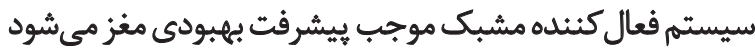

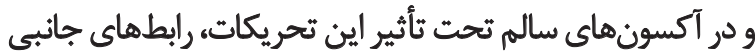

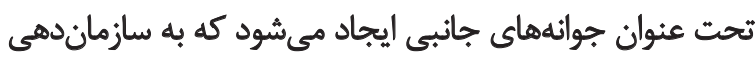

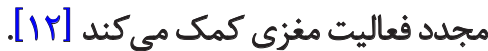
يكى از روشهايى كه به بهببود عملكرد مغز و ساير سيستمهاي

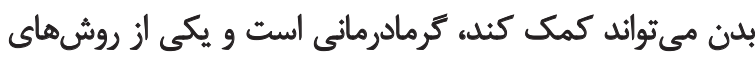

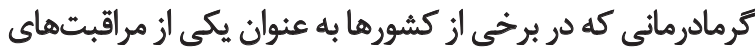

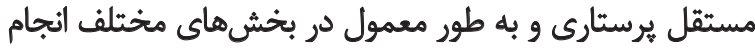

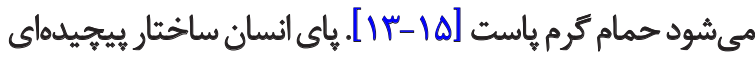

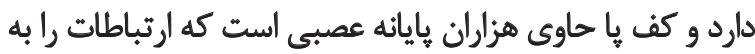

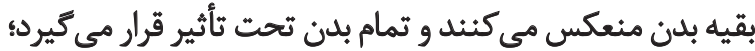

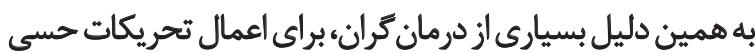

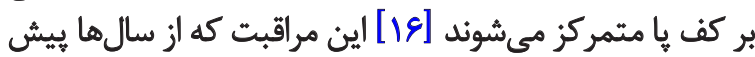

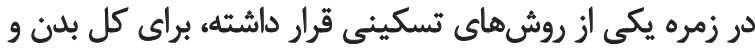

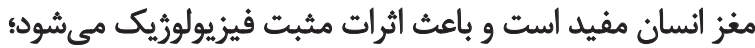

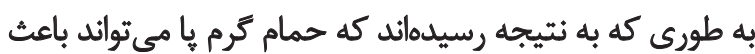

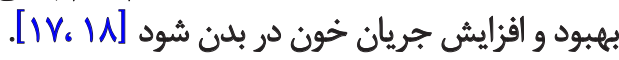
حمام گرم يا به عنوان يكى از روشهاي گرمادرمانى، از جمله

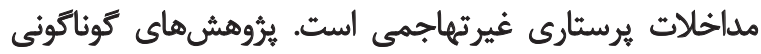

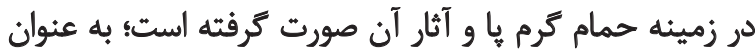

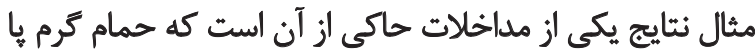

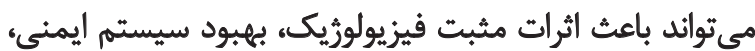

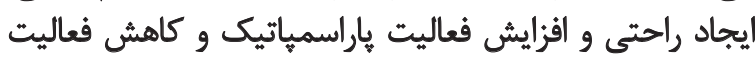

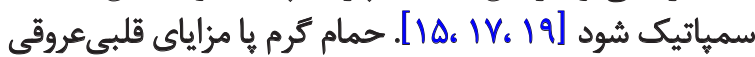

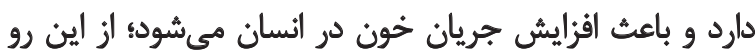

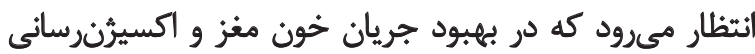

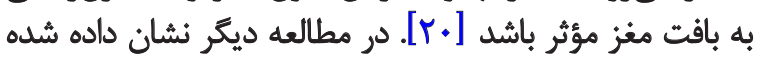


طول زمان نسبت به بررسى آن در نقطهاي از زمان ارزش بيشترى

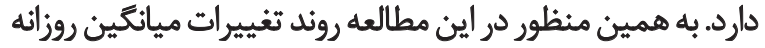

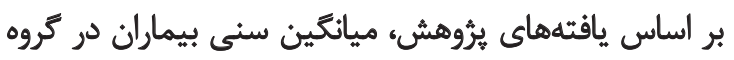

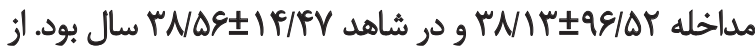

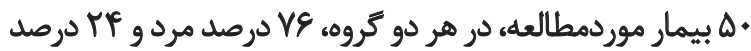

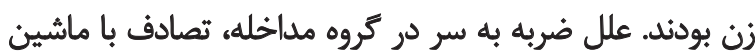

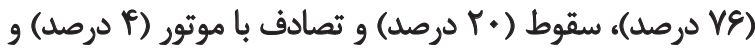

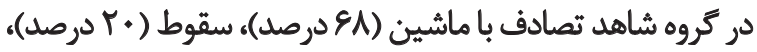

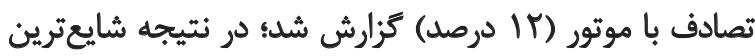

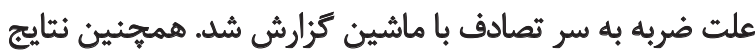

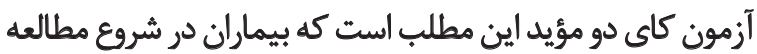

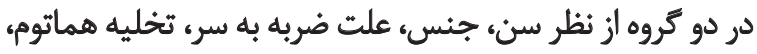

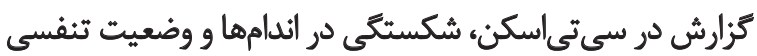

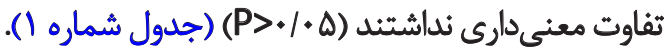

ميانكين سطح هوشيارى بيماران در شروع مطالعه در روز اول

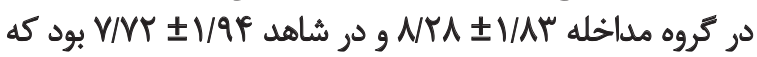

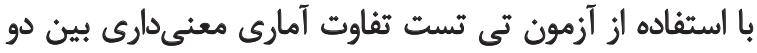

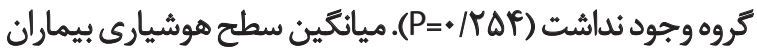

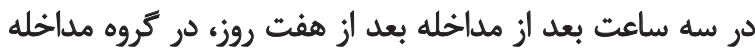

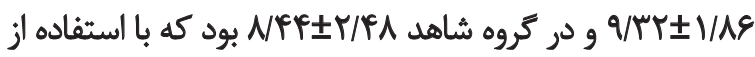

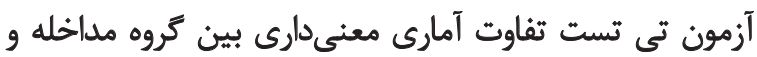

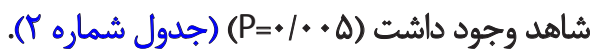

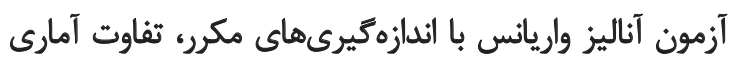

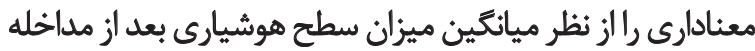

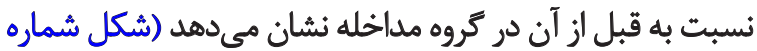

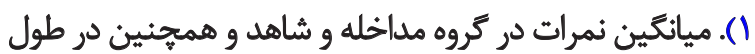

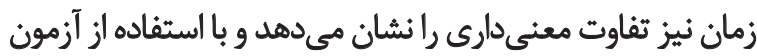

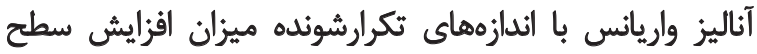

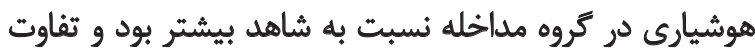

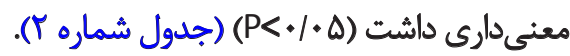

\section{بحث ونثيجنه كيرى}

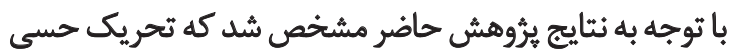

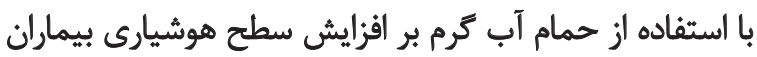

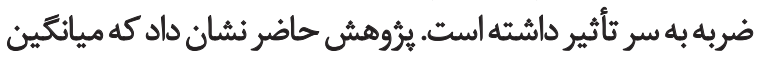

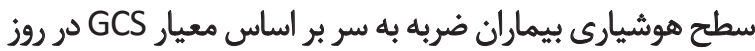

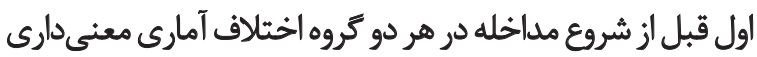

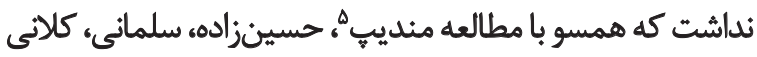

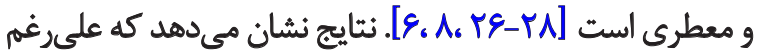

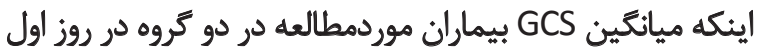

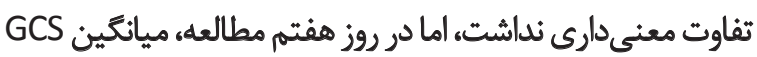
در كروه مداخله نسبت به تروه شاهد بالاتر بود.
نمره هوشيارى بيماران در گروه مداخله و شاهد طنى هلى هفت روز

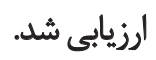

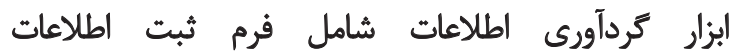

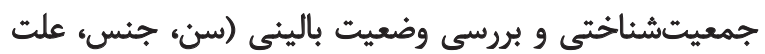

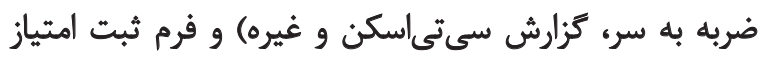

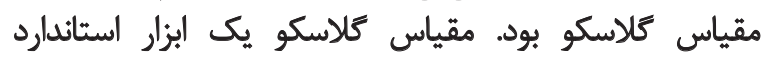

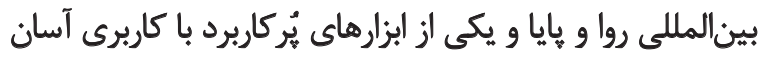

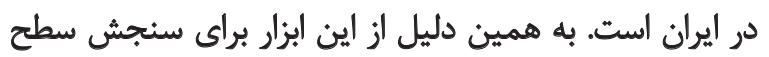

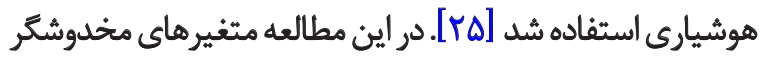

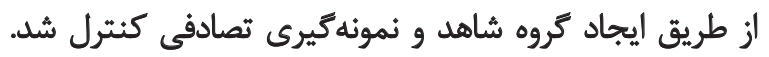

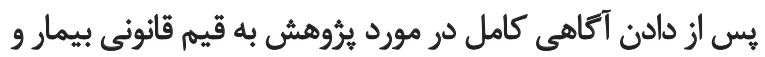
كرفتن رضايت آكاهائه نمونه كاميرى آغاز شورد بروش

ابتدا تخصيص تصادفى نمونهها براى دو تروه شاهد و و مداخله

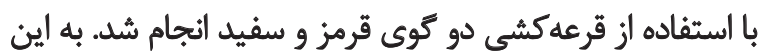

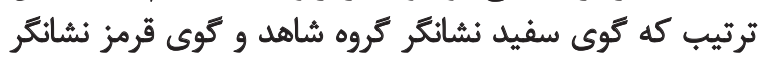

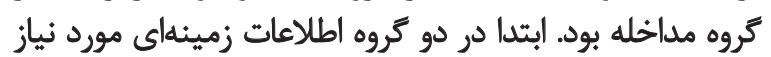

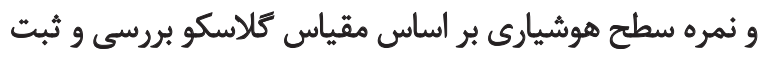

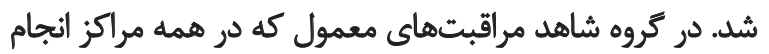

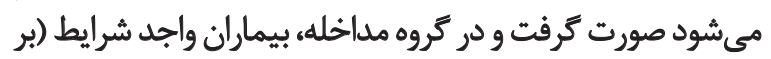

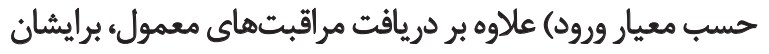

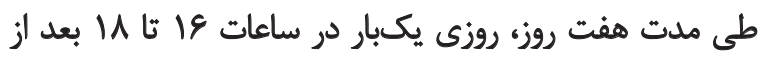

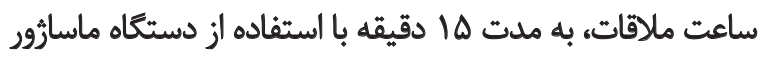

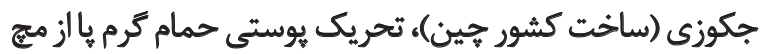

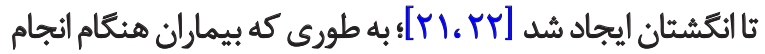

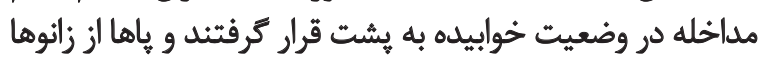

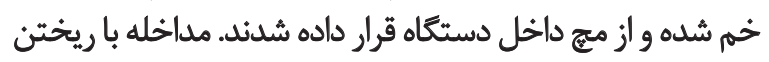

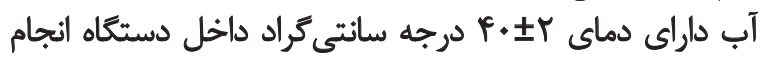

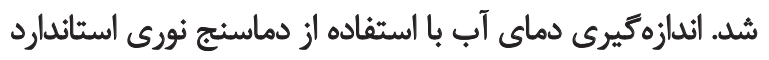

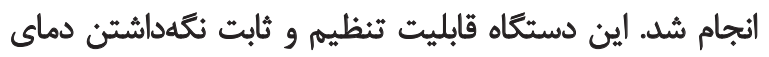

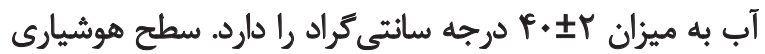

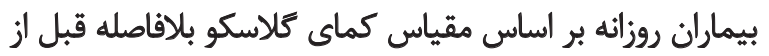

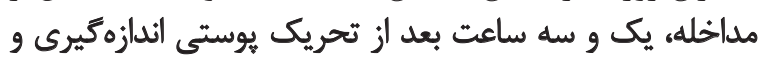

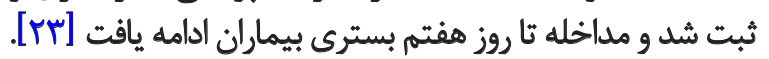

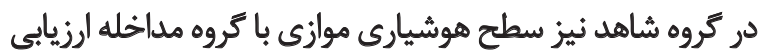

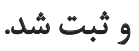

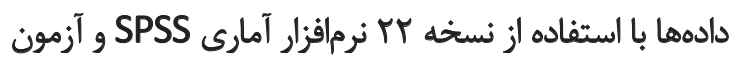

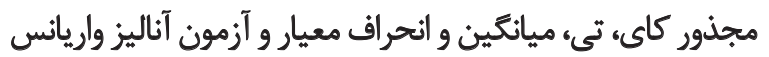

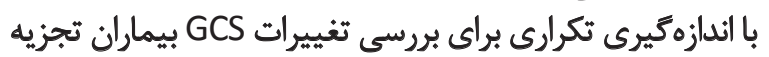

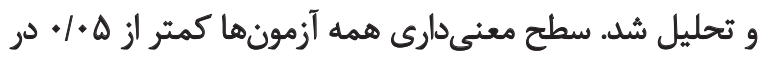

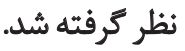


جدول ا.ويرّكى هاي بالينى نمونهاي موردبررسى

\begin{tabular}{|c|c|c|c|c|}
\hline سطح معنى دارى & شاهد & مداخله & \multicolumn{2}{|c|}{ اطلاعات جمعيتشناختى و باليثى } \\
\hline.$/ 9 \Delta F^{*}$ & WNAF士IF/FY & rNIrEQE/AT & \multicolumn{2}{|c|}{ ميائكين سن (سال) } \\
\hline \multirow{2}{*}{$.194 r^{* *}$} & 19 & 19 & مرد & \multirow{2}{*}{ جنسيت } \\
\hline & 8 & 8 & ن & \\
\hline \multirow{3}{*}{$+191 \%=0$} & IV & 19 & تصادف با هاشين & \multirow{3}{*}{ علث ضربه به سر } \\
\hline & $\Delta$ & $\Delta$ & سقوط & \\
\hline & $r$ & 1 & تصادف با موتور & \\
\hline \multirow{2}{*}{.$(A) V^{* *}$} & 18 & iv & 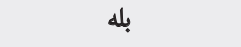 & \multirow{2}{*}{ عمل جراحى كرانيوتومى } \\
\hline & 9 & $A$ & خير & \\
\hline \multirow{2}{*}{$.1 \cdot 1 \theta^{* *}$} & $m$ & $M$ & 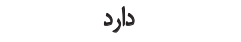 & \multirow{2}{*}{ مشكل تثنسى } \\
\hline & r & $\checkmark$ & ندارد & \\
\hline \multirow{2}{*}{$+1.94=$} & $\wedge$ & 11 & ل مارد & \multirow{2}{*}{ شكستكى اندام فوقانى } \\
\hline & IV & if & ندارد & \\
\hline \multirow{6}{*}{.$|\| V\rangle^{* *}$} & 9 & 11 & ت تكان مغزى & \multirow{6}{*}{ تشخْيص } \\
\hline & $\Delta$ & 8 & خُونريزى ناخل مغزى & \\
\hline & $r$ & $r$ & خونريزى زير عنكبوتيه & \\
\hline & f & $r$ & خونريزى زير سخت شامه & \\
\hline & $r$ & - & خونريزى اييدورال & \\
\hline & - & $r$ & الدم مغزى & \\
\hline
\end{tabular}

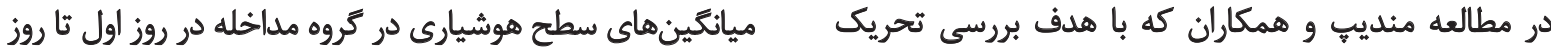

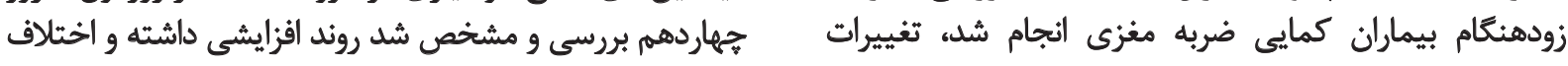

جدول †. مقايسه ميانكين سطح هوشيارى در زمانهاي بررسيى در كروههاي بيماران موردمطالعه

\begin{tabular}{|c|c|c|c|c|c|c|c|c|}
\hline \multicolumn{7}{|c|}{ ميانكيندانحراف معيار } & \multirow{2}{*}{ קوز } & \multirow{2}{*}{ كروه ها } \\
\hline روز هفتت & روز ششمي & روز هنجم & روز جهارم & روز سوم & روز دوم & روز اول & & \\
\hline Q/THEV/N & GratV/Ar & V.f \pm V/Ar & NASEI/QA & $N T T \pm 1 / Q \Delta$ & $N H Y \pm \mid / A 1$ & NTA \pm /Ar & هذاخله & \\
\hline NPS \pm T/TA & NYAET/Ar & $N F+ \pm T / N^{2}$ & $N \cdot N \pm r / I r$ & $N \cdot N \pm r / * 1$ & $V / \wedge f \pm Y / * \Delta$ & $V / V \pm I / 9 F$ & شاهد & ثبل از مداخله \\
\hline $1 * \circ 1^{*}$ & $.1 \cdot \mathrm{A}^{*}$ & $* 1 \cdot 1 *^{*}$ & $1+1^{*}$ & $\% n$ &.$/ 1 F A$ &.$/$ TQY & سطح معنى دارى & \\
\hline VTrEV/N & VRA土V/Ar & V.FIV/Ar & NF $\pm 1 / q \Delta$ & NTTII/QD & $N M T \pm / / A I$ & NRA \pm I/Ar & هذاخله & \\
\hline NPF \pm YT/R & NTA \pm T/AT & $M P F \pm T / M$ & $N \cdot N \pm Y / I Y$ & $N \cdot N \pm T / * r$ & $V / M \pm T / \cdot r$ & V/TTIV/ด & شاهد & بعد از مدائله \\
\hline $1 \cdots \infty^{*}$ & $.1 . .8 *$ & $.1 \cdot 10^{*}$ & $.1 \circ A^{*}$ & .100 &.$/ 109$ & - MAT & سطح معنى دارى & \\
\hline$M T \pm V / N$ & VRA \pm VIAT & $V \cdot N \pm 1 / N$ & NeTI/A9 & NTE $\pm 1 /$ M & $N M T \pm / / A I$ & NTAEI/AY & مداخله & \\
\hline NPTEYTR & NRA & NPPET/Yq & $N \cdot N \pm Y / I Y$ & $N \cdot N \pm T / * 1$ & $V / M \pm r / \cdot r$ & $V / M T \pm 1 / 9 \%$ & شاهد & "از ساعت بعلد \\
\hline $.1 * \Delta^{*}$ & $.10 .4 *$ & $.1 \cdot \cdot v^{*}$ & .1 .00 & $\cdot 1 \cdot \pi$ & $\cdot N \cdot r$ & - MAT & سطح معنى دارى & \\
\hline
\end{tabular}




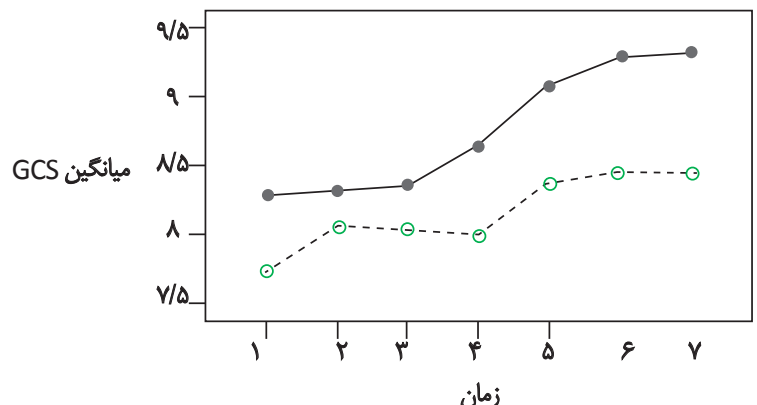

كرو مأو

هلأخله

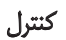

زمان

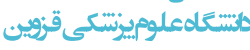

شكل ا. مقايسه ميانكينين نمره هوشيارى بيماران در دو كروه شاهد و مداخله در دوره بروسي

در اين يروهش افزايش سطح GCS بيماران كروه مداخله از براز

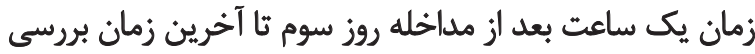

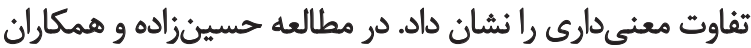

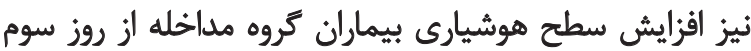

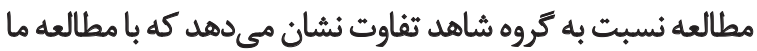

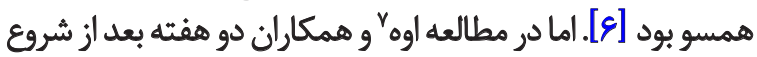

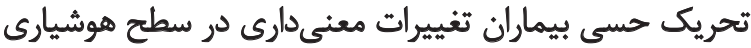

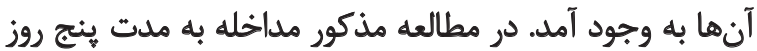

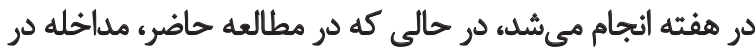

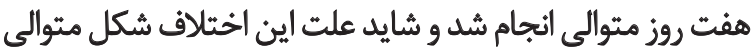
و مئقطعبودن مداخله است [ـr].

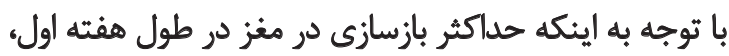

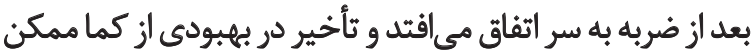

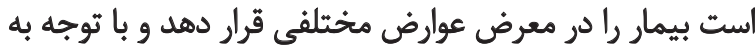

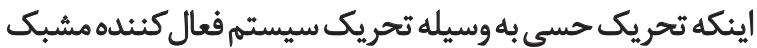

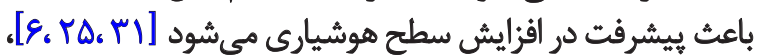

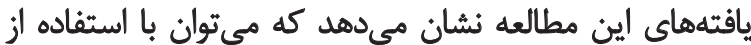

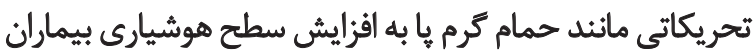

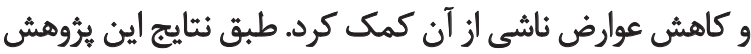

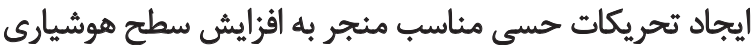

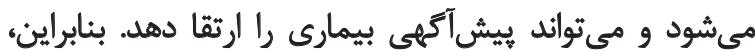

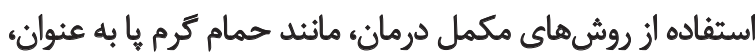

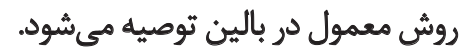

\section{مالامظاث الخالاقي \\ يبيروى أز اصول اخلاق يُؤوهش}

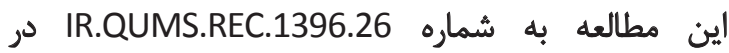

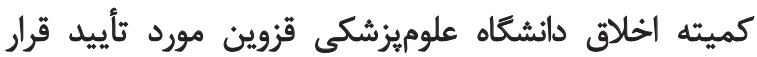

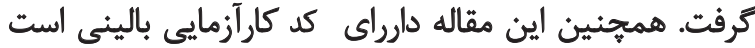

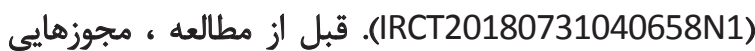

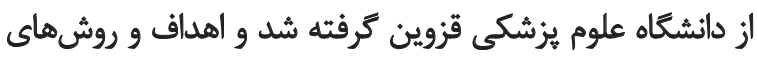

معنى دارى بين ميانكين روزهاى اول، هفتم و روز جهاردهم

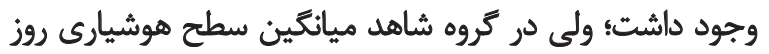

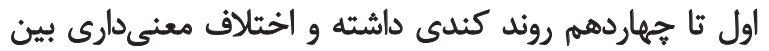

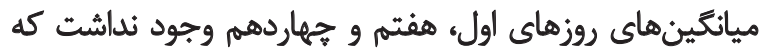

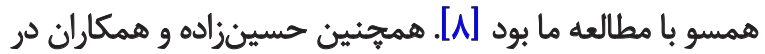

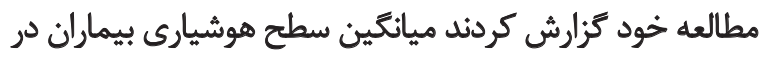

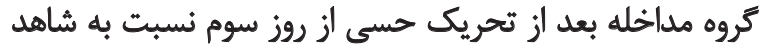

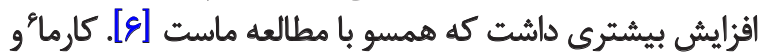

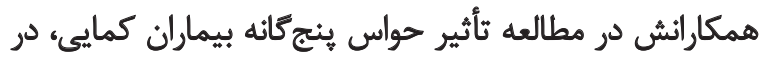

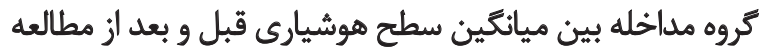

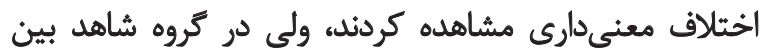

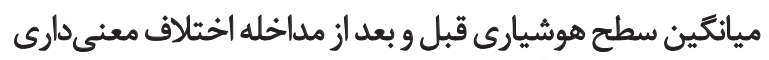

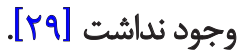

سلمانى و همكاران در مطالعه درباره اثر تحريك عاطفى خانواده

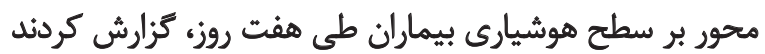

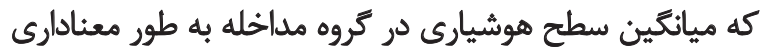

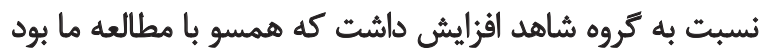

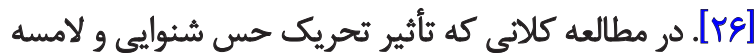

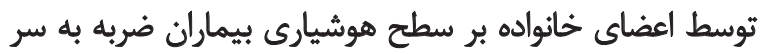

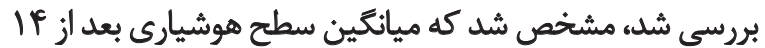

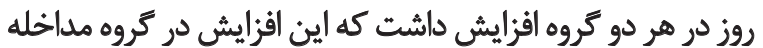

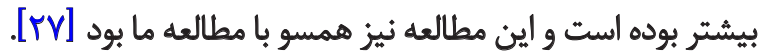

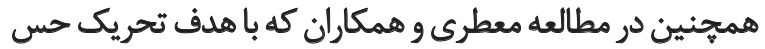

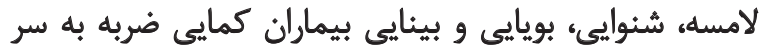

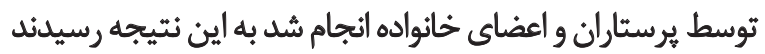

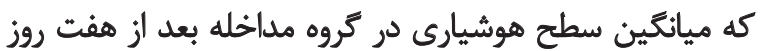

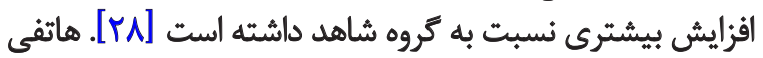

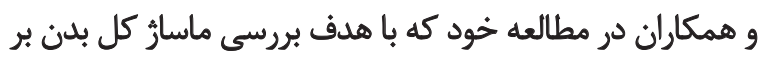

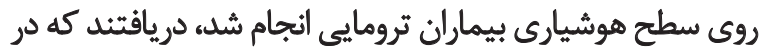

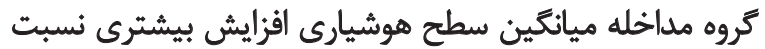

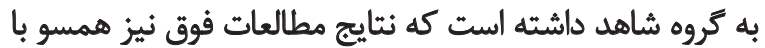

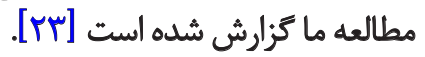


مطالعه به همراهان بيمار توضيح داده شد و رضايت آتاهانه

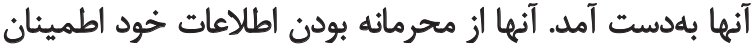

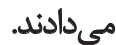

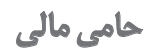

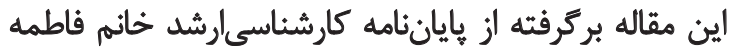

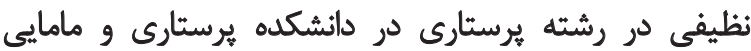

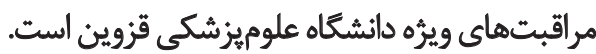

$$
\text { مشاركث نويسندكّان }
$$

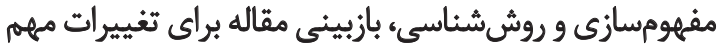

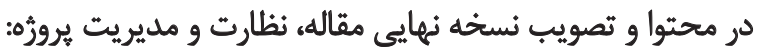

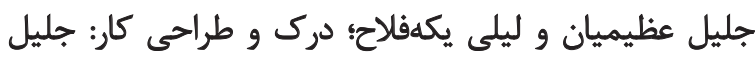

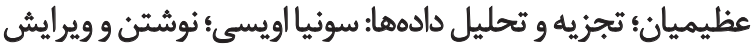

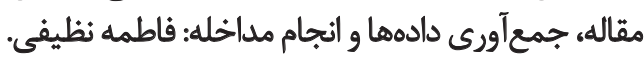

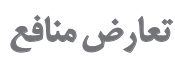

بنابر اظهار نويسندكان اين مقاله هيجّونه تعارض منافعى در ندارد.

$$
\text { تشكر و قدردافي }
$$

بدينوسيله از معاونت بروهشى دانشكاه علوميزشكى قزوين،

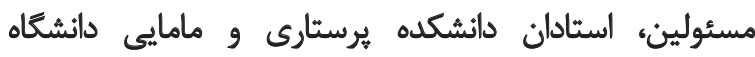

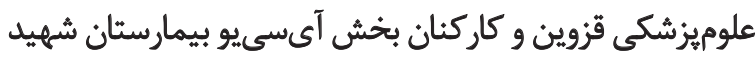

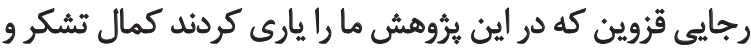

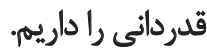




\section{References}

[1] Sina Trauma Research Center, TUMS-Center for Academic and Helth Policy. [Clinical guidelines for head trauma: (Triage, initial examination and treatment. $2^{\text {nd }}$ ed. Tehran: Puneh Publications; 2015. [In Persian] http://opac.nlai.ir/opac-prod/bibliographic/3858810

[2] Azimian J, Abdi M, Moradi M, Alipour Heidari M, Yekeh Fallah $L$, Sheikhi MR. The effect of foot massage on the consciousness levels in comatose patients with brain injury hospitalized in Intensive Care Unit (ICU): A randomised control trial. J Knowl Health Basic Med Sci. 2015; 10(3):25-30. [In Persian] [DOI:10.22100/jkh.v10i3.692]

[3] Faul M, Xu L, Wald MM, Coronado VG. Traumatic brain injury in the United States: Emergency department visits, hospitalizations and deaths 2002-2006 [Internet]. 2010 [Updated 2010 March]. Available from: http://www.cdc.gov/traumaticbraininjury/pdf/blue_book.pdf

[4] Iranian Legal Medicine Organization. Statistics of deaths and injuries caused by traffic accidents in 2015. [Internet]. 2015 [Updated 2015]. [In Persian] Available from: https://cutt.ly/ryv8tZS

[5] Feizi A, Jerineshin $H$, Estabraghnia $H$, Mahoori A, Khalkhali HR, Sattari S. Study of correlation of Glasgow Coma Score and Bispectral Index in mild and moderate traumatic brain injuries. Nurs Midwifery J. 2011; 9(5):394-9. [In Persian] http://unmf. umsu.ac.ir/article-1-613-en.html

[6] Mahmoodi GR, Hosseinzadeh E, Vakili MA, Kazemnejad K, Mohammadi MR, Taziki MH, et al. The effect of voice auditory stimulation on the consciousness of the coma patients suffering from head injury. J Res Dev Nurs Midwifery. 2013; 10(1):1-9. [In Persian] http://goums.ac.ir/jgbfnm/article-1-311-en.html

[7] Giacino JT, Fins JJ, Laureys S, Schiff ND. Disorders of consciousness after acquired brain injury: The state of the science. Nat Rev Neurol. 2014; 10(2):99-114. [DOI:10.1038/nrneurol.2013.279] [PMID]

[8] Mandeep PK. Effectiveness of early intervention of coma arousal therapy in traumatic head injury patients. Int $J$ Head Neck Surg. 2012; 3(3):137-42. [DOI:10.5005/jp-journals-10001-1114]

[9] Li WJ. The effect of warm-water footbath on fatigue, sleep and quality of life of stroke patients. Paper presented at: 25th International Nursing Research Congress. 24-28 July 2014; Hong Kong. https://stti.confex.com/stti/congrs14/webprogram/Paper65024.html

[10] Davis A, Gimenez A. Cognitive-behavioral recovery in comatose patients following auditory sensory stimulation. J Neurosci Nurs. 2003; 35(4):202-9. [DOI:10.1097/01376517-20030800000006] [PMID]

[11] Goudarzi F, Basampoor Sh, Zakeri-Moghadam M, Faghihzadeh S, Rezaie F, Mohammadzadeh F. Changes in level of consciousness during auditory stimulation by familiar voice in comatose patients. Iran J Nurs. 2010; 23(63):43-50. [In Persian] http://ijn.iums.ac.ir/article-1-772-en.html

[12] Gerber C. Understanding and managing coma stimulation: Are we doing everything we can? Crit Care Nurs Q. 2005; 28(2):94108. [DOI:10.1097/00002727-200504000-00002] [PMID]
[13] Hu Q, Zhu W, Zhu Y, Zheng L, Hughson RL. Acute effects of warm footbath on arterial stiffness in healthy young and older women. Eur J Appl Physiol Occup Physiol. 2012; 112(4):12618. [DOI:10.1007/s00421-011-2066-1] [PMID]

[14] Miyazato K, Matsukawa K. Decreased cardiac parasympathetic nerve activity of pregnant women during foot baths. Jpn J Nurs Sci. 2010; 7(1):65-75. [DOI:10.1111/j.17427924.2010.00136.x] [PMID]

[15] Saeki Y, Nagai N, Hishinuma M. Effects of footbathing on autonomic nerve and immune function. Complement Ther Clin Pract. 2007; 13(3):158-65. [DOI:10.1016/j.ctcp.2006.12.006] [PMID]

[16] Weiss RC. Your career in physical medicine [M. Golchin, Persian trans.]. Divani A, editor. $2^{\text {nd }}$ ed. Tehran: Shahrab; 2014. http://opac.nlai.ir/opac-prod/bibliographic/1335650

[17] Yamamoto K, Aso Y, Nagata S, Kasugai K, Maeda S. Autonomic, neuro-immunological and psychological responses to wrapped warm footbaths-a pilot study. Complement Ther Clin Pract. 2008; 14(3):195-203. [DOI:10.1016/j.ctcp.2008.04.001] [PMID]

[18] Mizutani M, Shimasaki H, Kawamura K, Nakagawa M, Maeda $\mathrm{K}$, Hamaguchi $\mathrm{H}$, et al. Influence of dynamic foot exercise and a warm-water footbath on arterial distensibility. J Jpn Soc Balneol Climatol Phys Med. 2015; 78(3):237-43. [DOI:10.11390/ onki.78.237]

[19] Inoue S, Kaneko F, Okamura H. Assessment of the efficacy of footbaths as a means of improving the mental health of nurses: A preliminary report. J Health Sci Hiroshima Univ. 2011; 9(2):27-30. [DOI:10.15027/33587]

[20] Ohnari H. Effect of microbubble bathing of lower extremities on peripheral circulation. Bull Yamaguchi Med Sch. 2010; 57(34):25-32. http://petit.lib.yamaguchi-u.ac.jp/G0000006y2j2/ metadata/A050057000301

[21] Azimian J, Abbasali Madadi Z, Falahatpishe F, Alipour Heidari $M$. Effect of warm footbath on arteriovenous fistula puncturerelated pain in dialysis patients. J Qazvin Univ Med Sci. 2015; 18(6):39-45. [In Persian] http://journal.qums.ac.ir/article1-1767-en.html

[22] Hassanzadeh F, Hoseini T, Esmaily H, Ehsaee MR. Impact of familiar sensory stimulation on level of consciousness in patients with head injury in ICU. J North Khorasan Univ Med Sci. 2012; 4(1):121-33. [In Persian] [DOI:10.29252/jnkums.4.1.121]

[23] Hatefi M, Jaafarpour M, Khani A, Khajavikhan J, Kokhazade T. The effect of whole body massage on the process and physiological outcome of trauma ICU patients: A double-blind randomized clinical trial. J Clin Diagn Res. 2015; 9(6):UC05-UC08. [DOI:10.7860/JCDR/2015/12756.6096] [PMID] [PMCID]

[24] Vahedian-Azimi A, Ebadi A, Asghari Jafarabadi M, Saadat S, Ahmadi F. Effect of massage therapy on vital signs and GCS scores of ICU patients: A randomized controlled clinical trial. Trauma Mon. 2014; 19(3):e17031. [DOI:10.5812/traumamon.17031] [PMID] [PMCID]

[25] Sosnowski C, Ustik M. Early intervention: Coma stimulation in the intensive care unit. J Neurosci Nurs 1994; 26(6):336-41. [DOI:10.1097/01376517-199412000-00005] [PMID] 
[26] Salmani F, Mohammadi E, Rezvani M, Kazemnezhad A. The effects of family-centered affective stimulation on brain-injured comatose patients' level of consciousness: A randomized controlled trial. Int J Nurs Stud. 2017; 74:44-52. [DOI:10.1016/j. ijnurstu.2017.05.014] [PMID]

[27] Kalani Z, Pourkermanian P, Alimohammadi N. The effect of family guided visits on the level of consciousness in traumatic brain injury. J Biol Today's World. 2016; 5(5):86-90. [DOI:10.15412/J.JBTW.01050502]

[28] Moattari M, Alizadeh Shirazi F, Sharifi N, Zareh N. Effects of a sensory stimulation by nurses and families on level of cognitive function, and basic cognitive sensory recovery of comatose patients with severe traumatic brain injury: A randomized control trial. Trauma Mon. 2016; 21(4):e23531. [DOI:10.5812/ traumamon.23531] [PMID] [PMCID]

[29] Karma D, Rawat AK. Effect of stimulation in coma. Indian Pediatr. 2006; 43(10):856-60. [PMID]

[30] $\mathrm{Oh} \mathrm{H}$, Seo W. Sensory stimulation programme to improve recovery in comatose patients. J Clin Nurs. 2003; 12(3):394-404. [DOI:10.1046/j.1365-2702.2003.00750.x] [PMID]

[31] Urbenjaphol P, Jitpanya C, Khaoropthum S. Effects of the sensory stimulation program on recovery in unconscious patients with traumatic brain injury. J Neurosci Nurs. 2009; 41(3):E10-E6. [DOI:10.1097/JNN.0b013e3181a23e94] 
This Page Intentionally Left Blank 